\title{
A Combined Spectral-Parabolic Equation Approach for Propagation Prediction in Tunnels
}

\author{
B. Izquierdo, J. Alonso, S. Capdevila, and J. Romeu \\ AntennaLab, Dept. of Signal Theory and Communications (TSC), \\ Universitat Politècnica de Catalunya (UPC), Barcelona, Spain. \\ E-mail: \{izquierdo,javalde,capdevila,romeu\}@tsc.upc.edu
}

\section{Introduction}

Several techniques can be used to study electromagnetic propagation in tunnels. A combination of two of such techniques is presented in this paper to model propagation inside a rectangular tunnel. They are combined in order to maximize the benefits inherent to their theoretical principles and restrictions. Fields in the region close to the transmitter are obtained by means of spectral theory [1],[2], whereas in the far field the parabolic regimen is used [3],[4].

\section{Spectral and Parabolic Methods}

Propagation in the spectral domain $[1],[2]$ is easily computed with an algebraic multiplication. Let $z$ be the longitudinal tunnel axis discretized in $\Delta z$ steps so that $z=i \Delta z, i=1,2 \cdots N$ and $E(x, y, n)$ a known field distribution on the transversal plane $i=n$. Fields at $i=n+1$ can be computed multiplying the angular plane wave spectrum $A_{E}\left(k_{x}, k_{y}, n\right)$ with phasor $e^{-j k_{z} \Delta z}$. $A_{E}\left(k_{x}, k_{y}, z_{0}\right)$ and $E\left(x, y, z_{0}\right)$ are related via FFT. The basic propagation scheme is:

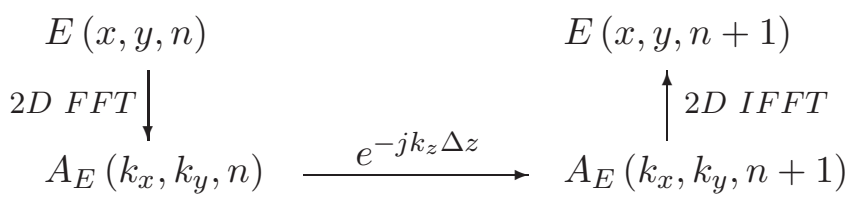

with $k_{z}=\sqrt{k_{0}^{2}-k_{x}^{2}-k_{y}^{2}}$. Boundary Conditions (BC) at $z=n+1$ are then enforced over $E(x, y, n+1)$. Propagation along the $z$-axis is achieved repeating the previous steps up to the end of the tunnel. The main features of this method are that the whole tunnel geometry is considered and information at any point of the tunnel cross-section can be easily incorporated in the model. Drawbacks arise when dealing with large meshes and long distances, even taking into account the efficiency of current FFT implementations. The computational burden increases and it may take unacceptable time to obtain results.

The Parabolic Equation (PE) - see [3],[4] - has been used for a long time in electromagnetic propagation modeling. Let $E=U e^{-j k_{z} z}, U$ being the approximate plane

\footnotetext{
${ }^{0}$ This work has been sponsored by IFERCAT (Infraestructures Ferroviaries de Catalunya) and was supported in part by the Spanish Interministerial Commission on Science and Technology (CICYT) under projects TEC2007-66698-C04-01/TCM and CONSOLIDER CSD2008-00068 and by the "Ministerio de Educacin y Ciencia" through the FPU fellowship program.
} 
wave solution at $i=n$, then $\mathrm{PE}$ can be expressed as:

$$
\frac{\partial U}{\partial z}=\frac{1}{2 j k_{0}}\left(\frac{\partial^{2} U}{\partial x^{2}}+\frac{\partial^{2} U}{\partial y^{2}}\right)
$$

After some manipulations and discretization $([5],[6])$ the Crank-Nicolson scheme is derived:

$$
\left(1-\frac{\Delta z}{2} A_{x}-\frac{\Delta z}{2} A_{y}\right) U^{n+1}=\left(1+\frac{\Delta z}{2} A_{x}+\frac{\Delta z}{2} A_{y}\right) U^{n}
$$

where $\Delta z$ may be set to a larger value than the one defined for the spectral method [4],[7], and $A_{x}$ and $A_{y}$ are the discretized 2nd-order differential operators in Eq.(1) $[5]$.

Although the solution at $z=n+1$ can be computed by matrix inversion at this point, the same limitations as in the spectral method in terms of computational cost arise. The Alternating Directional Implicit (ADI) [5],[6] overcomes this issue evolving the Crank-Nicolson scheme to a 2-step method that Peaceman and Rachford [8] expressed as:

$$
\begin{aligned}
& \left(1-\frac{\Delta z}{2} A_{x}\right) \tilde{U}^{n+\frac{1}{2}}=\left(1+\frac{\Delta z}{2} A_{y}\right) U^{n} \\
& \left(1-\frac{\Delta z}{2} A_{y}\right) U^{n+1}=\left(1+\frac{\Delta z}{2} A_{x}\right) \tilde{U}^{n+\frac{1}{2}}
\end{aligned}
$$

where $\tilde{U}^{n+\frac{1}{2}}$ is an intermediate virtual plane. Field computation is split in several 1D problems as the solution is reached decomposing the field line per line at both step. As tunnel walls are usually made of concrete or similar materials, Leontovich $\mathrm{BC}$ [9] are used. Although ADI is very efficient computationally, its validity is restricted to small propagation angles as the parabolic regimen is accurate only when propagation takes place predominantly in one direction. Hence, this method cannot be used in the source vicinity.

\section{The Combined Spectral-PE Approach}

The combination of both techniques is a good solution for the full range of distances: spectral methods are costly but provide an accurate field description near the transmitter and the ongoing reflections on the tunnel walls. When only the plane wave components that propagate along the longitudinal axis remain, the parabolic approach is used to obtain the solution in a much more efficient manner.

Propagation from the source plane begins with the spectral method. As the wavefront progresses, the amount of energy contained in the PE angular range of validity range is computed. This range is taken at $\pm 15^{\circ}[10]$. When it reaches a certain threshold, the propagation method is switched to ADI up to the end of the simulated tunnel. 
Measurements were made in the tunnel shown in Fig.1 [11]. It presents a quasirectangular shape. For simulations, an equivalent [12] rectangular 4.6m-high, 9.5mwide, $150 \mathrm{~m}$-long straight tunnel was considered. Fig.2 compares measurements and simulations at $5.8 \mathrm{GHz}$ using the above-described approach. A $\lambda / 2$ patch at $2 m$ height is placed centred at the tunnel axis. The patch was horizontally polarized for the first measurement set, and vertically polarized for the second set. At $30 \mathrm{~m}$ from the transmitter, a $99.5 \%$ of the energy is contained inside the $\pm 15^{\circ} \theta$ range for both cases and the spectral method shifts to the parabolic one. Although there are certain stretches where traces differ significantly, pathloss are similar overall. Inaccuracies are attributed to the tunnel modeling, since the simulation model considers only smooth walls without scatterers along the surface, as well as imprecise dimension selection of the equivalent rectangular tunnel.

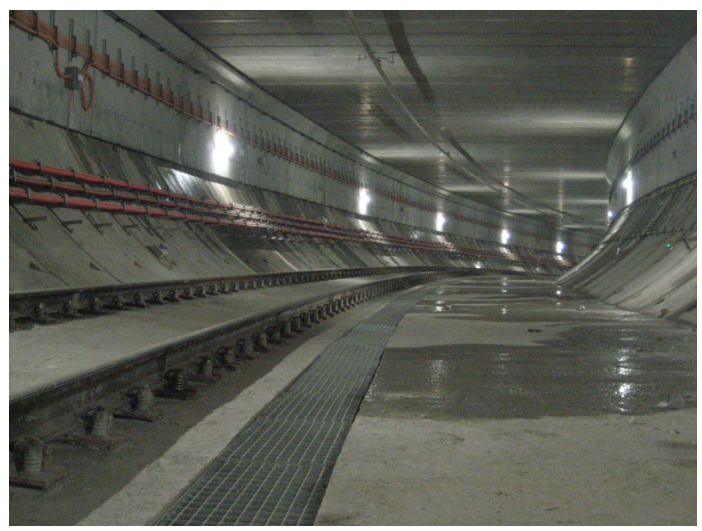

Figure 1: Measurement Tunnel.

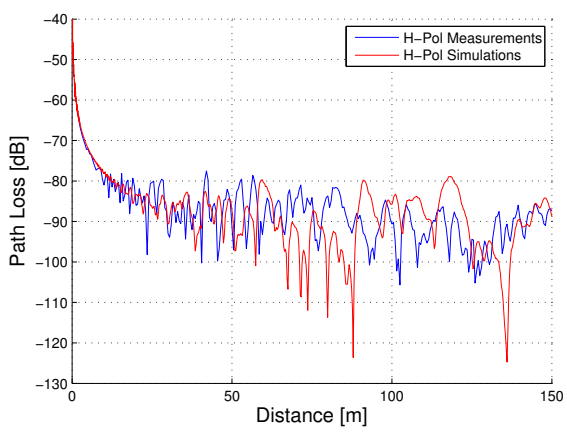

(a) Horizontal polarization

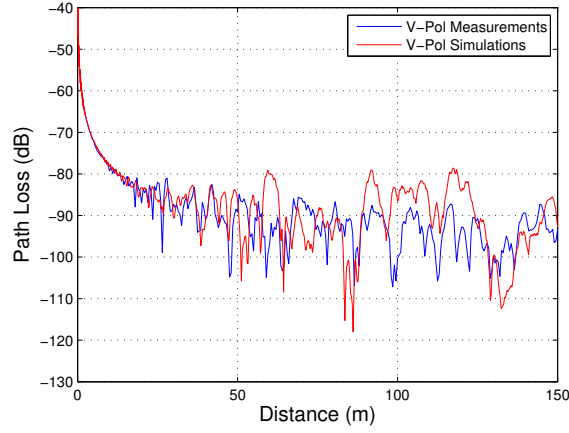

(b) Vertical polarization

Figure 2: Measurements and Simulations Comparison at $5.8 \mathrm{GHz}$

\section{References}

[1] J. Rius, A. Lozano, L. Jofre, and A. Cardama, "Spectral iterative algorithm for rcs computation in electrically large or intermediate perfectly conducting 
cavities," Antennas and Propagation, IEEE Transactions on, vol. 42, no. 6, pp. 790-797, 1994.

[2] B. Izquierdo, S. Capdevila, L. Jofre, and J. Romeu, "Evaluation of MIMO capacity in train tunnels," in Antennas and Propagation International Symposium, 2007 IEEE, S. Capdevila, Ed., 2007, pp. 1365-1368.

[3] M. Levy, Parabolic Equation Methods for Electromagnetic Wave Propagation. IEE Press, London, UK, 2000.

[4] C. Zelley and C. Constantinou, "A three-dimensional parabolic equation applied to VHF/UHF propagation over irregular terrain," Antennas and Propagation, IEEE Transactions on, vol. 47, no. 10, pp. 1586-1596, Oct 1999.

[5] J. C. Strikwerda, Finite Difference Schemes and Partial Differential Equations, 2nd ed. SIAM, 2004.

[6] R. Martelly and R. Janaswamy, "An ADI-PE Approach for Modeling Radio Transmission Loss in Tunnels," Antennas and Propagation, IEEE Transactions on, vol. 57, no. 6, pp. 1759-1770, June 2009.

[7] K. W. Morton and D. F. Mayers, Numerical Solution of Partial Differential Equations. Cambridge University Press, 2005.

[8] D. W. Peaceman and J. Rachford, H. H., "The numerical solution of parabolic and elliptic differential equations," Journal of the Society for Industrial and Applied Mathematics, vol. 3, no. 1, pp. 28-41, 1955.

[9] T. B. A. Senior, "Impedance boundary conditions for imperfectly conducting surfaces," Applied Scientific Research, Section B, vol. 8, no. 1, pp. 418-436, December 1969.

[10] D. Lee and S. McDaniel, "Ocean acoustic propagation by finite difference methods," Computers and Mathematics with Applications, vol. 14, no. 5, pp. 305423, 1987.

[11] J. Alonso, B. Izquierdo, S. Capdevila, and J. Romeu, "Preliminar Propagation and MIMO Experiments in Train Tunnels at 5.8GHz," in Antennas and Propagation Society International Symposium, 2009. APSURSI 2009. IEEE, 1-5 June 2009, pp. 1-4.

[12] J. Molina-Garcia-Pardo, M. Lienard, A. Nasr, and P. Degauque, "On the possibility of interpreting field variations and polarization in arched tunnels using a model for propagation in rectangular or circular tunnels," Antennas and Propagation, IEEE Transactions on, vol. 56, no. 4, pp. 1206-1211, April 2008. 